\title{
NORMAL CRITERION AND SHARED VALUE BY DERIVATIVES OF MEROMORPHIC FUNCTIONS
}

\author{
QIAN LU AND QILONG LIAO
}

\begin{abstract}
Let $\mathscr{F}$ be a family of meromorphic functions in a plane domain $D$. If for every function $f \in \mathscr{F}$, all of whose zeros have,at least,multiplicity $l$ and poles have, at least,multiplicity $p$, and for each pair functions $f$ and $g$ in $\mathscr{F}, f^{(k)}$ and $g^{(k)}$ share 1 in $D$, where $k, l$, and $p$ are three positive integer satisfying $\frac{k+1}{l}+\frac{1}{p} \leq 1$, then $\mathscr{F}$ is normal.
\end{abstract}

\section{Introduction and The Main Result}

W. K. Hayman[3] proved the following well-known result.

Theorem A. Let $f$ be a non-constant in the complex plane $C$, and $k$ be a fixed positive integer. Then either $f$ or $f^{(k)}-1$ has at least one zero. Moreover, if $f$ is transcendental, $f$ or $f^{(k)}-1$ has infinitely many zeros

Corresponding to Theorem A, Gu[2] proved the following famous result that is called Gu's Criterion, which is one of Hayman's [4] conjecture.

Theorem B. Let $k$ be a positive integer and $\mathscr{F}$ be a family of zero-free meromorphic functions in a complex domain $D$ such that $f^{(k)} \neq 1$ for each $f \in \mathscr{F}$, then $\mathscr{F}$ is normal.

Theorems B have been extensively and deeply studied. They focus on relaxing the conditions in Theorem B to that $f$ or $f^{(k)}-1$ has a zero.

Y. Wang and M. Fang [9] obtained the following result.

Theorem C.(See [9], Corollary 3.) Suppose that $n, k$ are two positive integers, $n \geq k+2$. Let $\mathscr{F}$ be a family of meromorphic function in plane domain $D$. If every function $f(z) \in \mathscr{F}$ has a zero of multiplicity $n$ at least, and $f^{(k)} \neq 1$, then $\mathscr{F}$ is normal in $D$.

Received October 6, 2011, accepted November 15, 2013.

2010 Mathematics Subject Classification. 30D35.

Key words and phrases. Meromorphic function, shared value, normal criterion.

Corresponding author: Qian Lu.

Supported by China Industrial Technology Development Program (B3120110001)and Application Basic Program of Science and Technology Department of SiChuan(07JY0029-06). 
Recently, M. J. Chang [5] generalizes Theorem B with only allowing that $f^{(k)}-1$ have zeros but restrcting their numbers. Y.T. Li[6] prove that the conclusion of Theorem B is also true under the condition that $f$ and $f^{\prime}-1$ may have a zero.

By the ideas of shared values, M.Fang and L.Zalcman[1], J. M. Qi and T.Y. Zhu[8] discuss the case that $f^{(k)}$ and $g^{(k)}$ share $a$ in $D$ and obtained

Theorem D.(see [1]) Suppose that $k$ is a positive integer and $a \neq 0$ is a finite complex numbers. Let $\mathscr{F}$ be a family of meromorphic function defined in a domain D. Iffor each pair of functions $f, g \in \mathscr{F}, f$ and $g$ share $0, f^{(k)}$ and $g^{(k)}$ share a IM in D, and the zeros of $f$ are of multiplicity $\geq k+2$, then $\mathscr{F}$ is normal in $D$.

Theorem E. (for the case $k \geq 2$, see [8], for the case $k=1$, see [7]) Let $\mathscr{F}$ be a family of meromorphic function defined in a domain $D$. Let $k$ be a positive integer and $a \neq 0$ is a finite complex numbers. If, for each $f \in \mathscr{F}$, all zeros of $f(z)$ are of multiplicity $\geq k+2$ and all zeros of $f^{(k)}$ are of multiplicity 2 at least and if, $f^{(k)}$ and $g^{(k)}$ share a IM in D for each pair of functions $f, g \in \mathscr{F}$, then $\mathscr{F}$ is normal in $D$.

In this paper, we obtain our main result as follow.

Theorem 1.1. Let $p, l$, and $k$ be three positive integers, $\frac{k+1}{l}+\frac{1}{p} \leq 1$, and $\mathscr{F}$ be a meromorphic function family in a plane domain $D$, all of whose zeros have,at least,multiplicity $l$ and poles have, at least, multiplicity $p$. If for each pair functions $f$ and $g$ in $\mathscr{F}, f^{(k)}$ and $g^{(k)}$ share 1 in $D$, then $\mathscr{F}$ is normal in $D$.

With Theorem 1.1, we immediately deduce the following corollary.

Corollary 1.2. Let $\mathscr{F}$ be a meromorphic function family in a plane domain D, all of whose zeros has, at least,multiplicity $2(k+1)$ and poles have, at least,multiplicity 2 , and $a \neq 0$ be $a$ finite complex number. If $f^{(k)}$ and $g^{(k)}$ share $a$ in $D$ for each pair functions $f$ and $g$ in $\mathscr{F}$, then $\mathscr{F}$ is normal in $D$, where $k$ be positive integer.

Corollary 1.3. Let $\mathscr{F}$ be a meromorphic function family in a plane domain $D$, all of whose zeros have,at least,multiplicity $k+2$ and poles has at least multiplicity $k+2$, and $a \neq 0$ be $a$ finite complex number. If $f^{(k)}$ and $g^{(k)}$ share $a$ in $D$ for each pair functions $f$ and $g$ in $\mathscr{F}$, then $\mathscr{F}$ is normal in $D$, where $k$ be positive integer.

With Corollary 1.3, we immediately the following result.

Corollary 1.4. Let $\mathscr{F}$ be a meromorphic function family in a plane domain $D$, and $k, n \geq k+2$ be positive integer and $a \neq 0$ be a finite complex number. If $\left(f^{n}\right)^{(k)}$ and $\left(g^{n}\right)^{(k)}$ share $a$ in $D$ for each pair functions $f$ and $g$ in $\mathscr{F}$, then $\mathscr{F}$ is normal in $D$.

Thereby, our Theorem 1.1 improves the result due to Y. T. Li and Yongxing Gu[7]. 


\section{Preliminary results}

Lemma 2.1. ([10]) Let $\mathscr{F}$ be a family of meromorphic functions on the unit disc $\Delta$, all of whose zeroes have multiplicity $p$ at least and poles have multiplicity $q$ at least. Let $\alpha$ be a real number satisfying $-p<\alpha<q$. Then $\mathscr{F}$ is not normal at a point $z_{0} \in \Delta$ if and only if there exist

(i) points $z_{n} \in \Delta, z_{n} \rightarrow z_{0}$;

(ii) functions $f_{n} \in \mathscr{F}$; and

(iii) positive numbers $\rho_{n} \rightarrow 0$

such that $\rho_{n}^{\alpha} f_{n}\left(z_{n}+\rho_{n} \xi\right)=g_{n}(\xi) \rightarrow g(\xi)$ spherically uniformly on each compact subset of $\mathbb{C}$, where $g(\xi)$ is a nonconstant meromorphic function satisfying the zeros of $g(\xi)$ are of multiplicities $p$ at least and the poles of $g(\xi)$ are of multiplicities $q$ at least. Moreover, the order of $g(\xi)$ is not greater than 2 .

Lemma 2.2. ([9]) Let $k$ be a positive integer, and $f(z)$ be a transcendental meromorphic function with finite orders in $\mathbb{C}$, whose zeros have multiplicity $k+2$ at least. Suppose that $f^{(k)} \neq 1$, then $f$ is a constant.

Lemma 2.3. ([4]) Let $f(z)$ be a transcendental meromorphic function in $\mathbb{C}$. If all zeros of $f(z)$ have multiplicity 3 at least, for any positive integer $k$, then $f^{(k)}$ assumes non-zero finite value infinitely often.

\section{Auxiliary lemmas}

Lemma 3.1. Let $p, l$, and $k$ be positive integers, $\frac{k+1}{l}+\frac{1}{p} \leq 1$, let $\varphi(z)$ be non-constant rational function, all of whose zeros has multiplicityl at least, and poles has multiplicity $p$ at least, then $\varphi^{(k)}(z)-1$ has two zeros at least.

Proof. Suppose not, then $\varphi^{(k)}(z)-1$ has one zeros at most. That is, either $\varphi^{(k)}(z)-1$ is freezero, or $\varphi^{(k)}(z)-1$ has exactly one zeros. From Lemma 2.2 , we have that $\varphi^{(k)}(z)-1$ has only one zeros. Set

$$
\varphi(z)=\frac{P(z)}{Q(z)}=A \frac{\left(z-\xi_{1}\right)^{n_{1}}\left(z-\xi_{2}\right)^{n_{2}} \cdots\left(z-\xi_{s}\right)^{n_{s}}}{\left(z-\xi_{1}\right)^{m_{1}}\left(z-\xi_{2}\right)^{m_{2}} \cdots\left(z-\xi_{s}\right)^{m_{t}}},
$$

where $P(z)$ and $Q(z)$ are co-prime polynomials, that is $(P(z), Q(z))=1, n_{i} \geq l(i=1,2, \cdots, s)$, and $m_{j} \geq p(j=1,2, \cdots, t) . \quad n=\sum_{i=1}^{s} n_{i}$ and $m=\sum_{j=1}^{t} m_{j}$ are the degree of $\operatorname{deg}(P(z))$ and $P(z)$,respectively. Moreover, denote $\omega_{P}$ as a product of factors $z-\xi_{i}$ corresponding to distinct zeros $z=\xi_{i}$ of polynomials $P$, and $d_{P}$ as a logithom derivative of $Q(z)$.

$$
\omega_{P}=\left(z-\xi_{1}\right)\left(z-\xi_{2}\right) \cdots\left(z-\xi_{s}\right)
$$




$$
\begin{aligned}
& d_{P}=\frac{P^{\prime}}{P}=\frac{n_{1}}{z-\xi_{1}}+\frac{n_{2}}{z-\xi_{2}}+\cdots+\frac{n_{s}}{z-\xi_{s}}, \\
& \omega_{Q}=\left(z-z_{1}\right)\left(z-z_{2}\right) \cdots\left(z-z_{s}\right), \\
& d_{Q}=\frac{Q^{\prime}}{Q}=\frac{m_{1}}{z-z_{1}}+\frac{m_{2}}{z-z_{2}}+\cdots+\frac{m_{s}}{z-z_{s}} .
\end{aligned}
$$

By mathematical induction, we have

$$
Q^{(k)}= \begin{cases}Q\left[\left(d_{Q}\right)^{k}+\sum_{p=2}^{k-1} \Psi_{p}\left(d_{Q}, d_{Q}^{\prime}, \cdots, d_{Q}^{(k-1)}\right)+d_{Q}^{(k-1)}\right], & k \leq m, \\ 0, & k>m .\end{cases}
$$

where $\Psi_{p}$ is a homogeneous differential polynomials with order $p$ and weight $k$ about $d_{Q}$, and

$$
\Psi_{p}\left(d_{Q}, d_{Q}^{\prime}, \cdots, d_{Q}^{(k-1)}\right)=\sum_{j} a_{p j}\left(d_{Q}\right)^{t_{p j 0}}\left(d_{Q}^{\prime}\right)^{t_{p j 1}} \cdots\left(d_{Q}^{(k-1)}\right)^{t_{p j, k-1}}, k \leq m
$$

satisfying $t_{p j 0}+t_{p j 1}+\cdots+t_{p j, k-1}=p$, and $t_{p j 0}+2 t_{p j 1}+\cdots+k t_{p j, k-1}=k$.

Set

$$
g_{k}\left(\psi_{1}, \psi_{2}, \cdots, \psi_{k}\right)=\psi_{1}^{k}+\sum_{p=2}^{k-1} \Psi_{p}\left(\psi_{1}, \psi_{2}, \cdots, \psi_{k}\right)+\psi_{k}, k \leq m,
$$

where $\psi_{1}=\omega_{Q} d_{Q}, \psi_{2}=\omega_{Q}^{2} d_{Q}^{\prime}, \cdots, \psi_{k}=\omega_{Q}^{k} d_{Q}^{(k-1)}$.

From (3.2),(3.3) and (3.4), we have

$$
Q^{(k)}=Q \omega_{Q}^{-k} \cdot g_{k}\left(\psi_{1}, \psi_{2}, \cdots, \psi_{k}\right), k \leq m,
$$

Since $\left(z-z_{j}, \psi_{r}\right)=1(j=1,2, \cdots, t ; r=1,2, \cdots, k)$, we have $\left(z-z_{j}, g_{k}\left(\psi_{1}, \psi_{2}, \cdots, \psi_{k}\right)\right)=1$. Thus

$$
\operatorname{deg}\left[g_{k}\left(\psi_{1}, \psi_{2}, \cdots, \psi_{k}\right)\right]=k(t-1),
$$

On the other hand, also by mathematical induction, we deduce that

$$
\left(\frac{1}{Q}\right)^{(k)}=\frac{(-1)^{k} k !\left(Q^{\prime}\right)^{k}}{Q^{k+1}}+\sum_{p=3}^{k} C_{p} \frac{H_{p-1}\left(Q^{\prime}, Q^{\prime \prime}, \cdots, Q^{(k-1)}\right)}{Q^{p}}-\frac{Q^{(k)}}{Q^{2}},
$$

where $H_{p}\left(Q^{\prime}, Q^{\prime \prime}, \cdots, Q^{(k-1)}\right)$ is a homogeneous differential polynomials about $Q$ with order $p$ and weight $k$ satisfying

$$
H_{p}\left(Q^{\prime}, Q^{\prime \prime}, \cdots, Q^{(k-1)}\right)=\sum_{j} b_{p j}\left(Q^{\prime}\right)^{n_{p j 1}}\left(Q^{\prime \prime}\right)^{n_{p j 2}} \cdots\left(Q^{(k-1)}\right)^{n_{p j, k-1}},
$$

where $k \leq m, n_{p j 1}+n_{p j 2}+\cdots+n_{p j, k-1}=p$ and $n_{p j 1}+2 n_{p j 2}+\cdots+(k-1) n_{p j, k-1}=k$. 
If $k>m$,

$$
H_{p}\left(Q^{\prime}, Q^{\prime \prime}, \cdots, Q^{(k-1)}\right)=\sum_{j} b_{p j}\left(Q^{\prime}\right)^{n_{p j 1}}\left(Q^{\prime \prime}\right)^{n_{p j 2}} \cdots\left(Q^{(m-1)}\right)^{n_{p j, m-1}},
$$

where $n_{p j 1}+n_{p j 2}+\cdots+n_{p j, m-1}=p$ and $n_{p j 1}+2 n_{p j 2}+\cdots+(m-1) n_{p j, m-1}=k$. It would be looked as $n_{p j, m+1}=n_{p j, m+2}=\cdots=n_{p j, k-1}=0$ in (3.8).

By (3.5), we have

$$
\begin{aligned}
& H_{p}\left(Q^{\prime}, Q^{\prime \prime}, \cdots, Q^{(k-1)}\right)=\sum_{j} b_{p j}\left(Q^{\prime}\right)^{n_{p j 1}}\left(Q^{\prime \prime}\right)^{n_{p j 2}} \cdots\left(Q^{(k-1)}\right)^{n_{p j, k-1}}, \\
= & \sum_{j} b_{p j}\left(Q \omega_{Q}^{-1} g_{1}\right)^{n_{p j 1}}\left(Q \omega_{Q}^{-2} g_{2}\right)^{n_{p j 2}} \cdots\left(Q \omega_{Q}^{-k} g_{k-1}\right)^{n_{p j, k-1}}, \\
= & Q^{p} \omega_{Q}^{-k} \sum_{j} b_{p j}\left(g_{1}\right)^{n_{p j 1}}\left(g_{2}\right)^{n_{p j 2}} \cdots\left(g_{k-1}\right)^{n_{p j, k-1}}
\end{aligned}
$$

Thereby, from (3.7) and (3.10), it follows

$$
Q^{k+1}\left(\frac{1}{Q}\right)^{(k)}=Q^{k} \omega_{Q}^{-k} \cdot \Phi_{k}\left(\psi_{1}, \psi_{2}, \cdots, \psi_{k}\right),
$$

when $k \leq m$

$$
\Phi_{k}\left(\psi_{1}, \cdots, \psi_{k}\right)=(-1)^{k} k !\left(\psi_{1}\right)^{k}+\sum_{p=3}^{k} C_{p} Q^{k-p+1} H_{p-1}\left(\psi_{1}, \cdots, \psi_{k}\right)-Q^{k-1} \psi_{k},
$$

and $k>m$,

$$
\Phi_{k}\left(\psi_{1}, \cdots, \psi_{k}\right)=(-1)^{k} k !\left(\psi_{1}\right)^{k}+\sum_{p=3}^{k} C_{p} Q^{k-p+1} H_{p-1}\left(\psi_{1}, \cdots, \psi_{m}\right),
$$

and furthermore $\operatorname{deg}\left[\Phi_{k}\left(\psi_{1}, \psi_{2}, \cdots, \psi_{k}\right)\right]=k(t-1)$.

Similarly, set $\phi_{1}=\omega_{P} d_{P}, \phi_{2}=\omega_{P}^{2} d_{P}^{\prime}, \cdots, \phi_{k}=\omega_{P}^{k} d_{P}^{(k-1)}$, and

$$
f_{k}\left(\phi_{1}, \phi_{2}, \cdots, \phi_{k}\right)=\phi_{1}^{k}+\sum_{p=2}^{k} \Psi_{p}\left(\phi_{1}, \phi_{2}, \cdots, \phi_{k}\right)+\phi_{k} \text {, }
$$

So from (3.5), we have

$$
P^{(k)}=P \omega_{P}^{-k} \cdot f_{k}\left(\phi_{1}, \phi_{2}, \cdots, \phi_{k}\right),
$$

Similarly,

$$
\operatorname{deg}\left[f_{k}\left(\psi_{1}, \psi_{2}, \cdots, \psi_{k}\right)\right]=k(s-1) .
$$

Thus, $\left(z-\xi_{i}, \phi_{r}\right)=1(i=1,2, \cdots, s ; r=1,2, \cdots, k)$, and we also have $\left(z-\xi_{i}, f_{k}\left(\phi_{1}, \phi_{2}, \cdots, \phi_{k}\right)\right)=1$ 
Therefore, it deduce that

$$
\begin{aligned}
\left(\frac{P}{Q}\right)^{(k)} & =\sum_{r=0}^{k}\left(\begin{array}{l}
k \\
r
\end{array}\right)\left(\frac{1}{Q}\right)^{(r)} P^{(k-r)}, \\
& =\frac{\sum_{r=0}^{k}\left(\begin{array}{l}
k \\
r
\end{array}\right)\left[Q^{r+1}\left(\frac{1}{Q}\right)^{(r)}\right] \cdot Q^{k-r} \cdot P^{(k-r)}}{Q^{k+1}} \equiv \frac{R(z)}{Q^{k+1}}, \\
& =\frac{\sum_{r=0}^{k}\left(\begin{array}{l}
k \\
r
\end{array}\right)\left[\left(Q \omega_{Q}^{-1}\right)^{r} \Phi_{r}\right] \cdot Q^{k-r} \cdot P \omega_{P}^{-(k-r)} f_{k-r}}{Q^{k+1}}, \\
& =\frac{P \omega_{P}^{-k}}{Q \omega_{Q}^{k}} \sum_{r=0}^{k}\left(\begin{array}{l}
k \\
r
\end{array}\right) \omega_{Q}^{k-r} \Phi_{r} \cdot \omega_{P}^{r} f_{k-r},
\end{aligned}
$$

Set

$$
h_{k}(z)=\sum_{r=0}^{k}\left(\begin{array}{l}
k \\
r
\end{array}\right) \omega_{Q}^{k-r} \Phi_{r} \cdot \omega_{P}^{r} f_{k-r}
$$

we have $\left(z-\xi_{i}, h(z)\right)=1(i=1,2, \cdots, s),\left(z-z_{j}, h(z)\right)=1(j=1,2, \cdots, t), \operatorname{deg}\left[h_{k}(z)\right] \leq k(s+t-1)$, and

$$
\left(\frac{P}{Q}\right)^{(k)}=\frac{P \omega_{P}^{-k}}{Q \omega_{Q}^{k}} h_{k}(z)
$$

Set

$$
\begin{aligned}
\tau_{1} & =\operatorname{deg}\left(P \omega_{P}^{-k} h_{k}(z)\right), \\
\tau_{2} & =\operatorname{deg}\left(Q \omega_{Q}^{k}\right), \\
N & =\max \left(\tau_{1}, \tau_{2}\right),
\end{aligned}
$$

then

$$
\begin{aligned}
& \left(\frac{P}{Q}\right)^{(k)}-1=A \frac{\left(z-z_{0}\right)^{N}}{Q \omega_{Q}^{k}} \\
& \left(\frac{P}{Q}\right)^{(k+1)}=A \frac{\left(z-z_{0}\right)^{N-1} g(z)}{Q \omega_{Q}^{k+1}}
\end{aligned}
$$

where $g(z)=N \omega_{Q}-\left(z-z_{0}\right)\left(g_{1}+k g_{0}\right)$, and $g_{0}=\omega_{Q} d_{\omega_{Q}}=\omega_{Q}\left(\frac{1}{z-z_{1}}+\frac{1}{z-z_{2}}+\cdots+\frac{1}{z-z_{t}}\right)$.

On the other hand, from (3.19) we have

$$
\left(\frac{P}{Q}\right)^{(k+1)}=\frac{P \omega_{P}^{-k-1}}{Q \omega_{Q}^{k+1}} h_{k+1}(z), \quad \operatorname{deg}\left[h_{k+1}\right] \leq(k+1)(s+t-1) .
$$

In the following, we distinguish three cases. 
Case 1. $n<m$. We have

$$
\tau_{1}-\tau_{2}=n-m-k(s+t)+\operatorname{deg}\left(h_{k}\right)<0 .
$$

Then, $N=\tau_{2}=m+k t$ and $\operatorname{deg}[g(z)]=t$. From (3.21) and (3.22), we have $N-1 \leq d e g\left[h_{k+1}\right]$ and $n-s(k+1) \leq \operatorname{deg}[g(z)]$. That is, $m \leq(k+1) s+t-k$ and $n \leq(k+1) s+t$. Since $\varphi(z)$ has a zero of multiplicity $l$ at least and a pole of multiplicity $p$ at least, we have $p t \leq m \leq(k+1) s+t-k$ and $l s \leq n \leq(k+1) s+t$. Thus, $\frac{k+1}{l}+\frac{1}{p}>1$, which contradicts $\frac{k+1}{l}+\frac{1}{p} \leq 1$.

Case 2. $n=m$. We have $\tau_{1}<\tau_{2}$. Thus, $N=m+k t$ and $\operatorname{deg}[g(z)] \leq t-1$. Similar to Case 1 , it deduce that $\frac{k+1}{l}+\frac{1}{p}>1$, a contradiction.

Case 3. $n>m$. We divide it into two subcase.

Subcase $3.1 n \geq m+k$. Set

$$
\frac{P(z)}{Q(z)}=P_{n-m}(z)+\frac{R_{\tau}}{Q(z)},
$$

then

$$
\begin{aligned}
{\left[\frac{P(z)}{Q(z)}\right]^{(k)}-1 } & =P_{n-m}^{(k)}(z)+\left[\frac{R_{\tau}(z)}{Q(z)}\right]^{(k)}-1=P_{n-m}^{(k)}(z)+\frac{R(z)}{Q^{k+1}(z)}-1 \\
& =\frac{P_{n-m}^{(k)}(z) Q^{k+1}(z)+R(z)-Q^{k+1}(z)}{Q^{k+1}(z)} .
\end{aligned}
$$

Since $\operatorname{deg}[R(z)]=r+k(m-1)<m(k+1) \leq \operatorname{deg}\left[P_{n-m}^{(k)}(z) Q^{k+1}(z)\right]$, by (3.20) we obtain $N=$ $n+k(t-1)$. From (3.21) and (3.22), we get $n \leq(k+1) s+t$. Since $n \geq l s$ and $n \geq p t+k$, we can obtain $\frac{k+1}{l}+\frac{1}{p}>1$, a contradiction.

Subcase 3.2. $n<m+k$. We also distinguish two subcases. Write

$$
\frac{P(z)}{Q(z)}=P_{n-m}(z)+\frac{R_{\tau}}{Q(z)} .
$$

Then

$$
\begin{aligned}
{\left[\frac{P(z)}{Q(z)}\right]^{(k)}-1 } & =P_{n-m}^{(k)}(z)+\left[\frac{R_{\tau}(z)}{Q(z)}\right]^{(k)}-1 \\
& =P_{n-m}^{(k)}(z)+\frac{R(z)}{Q^{k+1}(z)}-1=\frac{R(z)-Q^{k+1}(z)}{Q^{k+1}(z)} .
\end{aligned}
$$

Since $\operatorname{deg}[R(z)]=r+k(m-1)<m(k+1), \operatorname{deg}\left[R(z)-Q^{k+1}(z)\right]=m(k+1)$. Therefore, from (3.20),we have $N=m+k t$. Applying (3.21) and (3.22), we get $m+k t-1 \leq(k+1)(s+t-1)$ and $n \leq(k+1) s+t$. Noting $n \geq l s$ and $m \geq p t$, we can obtain $\frac{k+1}{l}+\frac{1}{p}>1$, a contradiction. 


\section{Proof of Theorem 1.1}

Suppose that $\mathscr{F}$ is not normal in $D$, that is, there exists one point $z_{0} \in D$ such that $\mathscr{F}$ is not normal at point $z_{0}$. Without loss of generality we assume that $z_{0}=0$. By Lemma 2.1, there exists a point sequence $\left\{z_{n}\right\}, z_{n} \in D, z_{n} \rightarrow z_{0}$, functions $f_{n} \in \mathscr{F}$, and positive numbers $\rho_{n} \rightarrow 0$ such that

$$
\varphi_{j}(\xi)=\rho_{j}^{-k} f_{j}\left(z_{j}+\rho_{j} \xi\right) \Rightarrow \varphi(\xi)
$$

spherically uniformly on each compact subset of $\mathbb{C}$, where $\varphi(\xi)$ is a nonconstant meromorphic function with order $\leq 2$, all of whose poles are of multiplicities $p$ at least and zeroes are of multiplicities $l$ at least.

From (4.1), we have

$$
\varphi_{j}^{(k)}(\xi)-1=f_{j}^{(k)}\left(z_{j}\right)-1 \Rightarrow \varphi^{(k)}(\xi)-1,
$$

also locally uniformly with respect to the spherical metric.

Obviously, $\varphi^{(k)} \not \equiv 1$, since otherwise $\varphi(z)=P_{k}(z)$ is a polynomial with a degree $k$. Thus, all zeros of $\varphi(z)$ are of multiplicity $k$ at most, a contradiction.

By Lemma 2.3, it follows that $\varphi^{(k)}-1$ has a zero at least. Claim that $\varphi^{(k)}-1$ has a unique zero $\xi=\xi_{0}$.

Indeed, if there exist two distinct zeros $\xi_{0}$ and $\xi_{0}^{*}, \xi_{0} \neq \xi_{0}^{*}$, then we choose a positive number $\delta>0$ such that a neighborhood $N\left(\xi_{0}, \delta\right)=\left\{\xi:\left|\xi-\xi_{0}\right|<\delta\right\}$ with radius $\delta$ of $\xi_{0}$ and a neighborhood $N\left(\xi_{0}^{*}, \delta\right)=\left\{\xi:\left|\xi-\xi_{0}^{*}\right|<\delta\right\}$ with radius $\delta$ of $\xi_{0}^{*}$ having no intersection, $N\left(\xi_{0}, \delta\right) \cap N\left(\xi_{0}^{*}, \delta\right)=$ $\phi$.

Since $\varphi^{(k)} \not \equiv 1$, from (4.2) and Hurwitz's theorem, there exists points $\xi_{j} \in N\left(\xi_{0}, \delta\right)$ and $\xi_{j}^{*} \in N\left(\xi_{0}^{*}, \delta\right)$ such that for sufficiently large $j$

$$
\begin{aligned}
& f_{j}^{(k)}\left(z_{j}+\rho_{j} \xi_{j}\right)-1=0, \\
& f_{j}^{(k)}\left(z_{j}+\rho_{j} \xi_{j}^{*}\right)-1=0 .
\end{aligned}
$$

According to the hypothesis that $f^{(k)}$ and $g^{(k)}$ share 1 in $D$ for each pair of $f$ and $g$ in $\mathscr{F}$, we know that for any integer $m$

$$
\begin{aligned}
& f_{m}^{(k)}\left(z_{j}+\rho_{j} \xi_{j}\right)-1=0, \\
& f_{m}^{(k)}\left(z_{j}+\rho_{j} \xi_{j}^{*}\right)-1=0 .
\end{aligned}
$$

Fixing $m$, and taking $j \rightarrow+\infty$, we deduce that

$$
f_{m}^{(k)}(0)-1=0
$$


Since the isolation of zeros for $f_{m}^{(k)}(z)-1$, for sufficiently large $j$, we have

$$
z_{j}+\rho_{j} \xi_{j}=0, \quad z_{j}+\rho_{j} \xi_{j}^{*}=0 .
$$

Thus,

$$
\xi_{j}=-\frac{z_{j}}{\rho_{j}}, \quad \xi_{j}^{*}=-\frac{z_{j}}{\rho_{j}} .
$$

That is, $\xi_{j}=\xi_{j}^{*} \in N\left(\xi_{0}, \delta\right) \cap N\left(\xi_{0}^{*}, \delta\right)$, this contradicts that $N\left(\xi_{0}, \delta\right) \cap N\left(\xi_{0}^{*}, \delta\right)=\phi$. And claim is proved.

From Lemma 2.3, it follows that $\varphi(z)$ must be a rational function. Also by Lemma 3.1, we deduce that $\varphi(z)$ has two zeros at least. This contradicts with previous claim that $\varphi(z)$ only has a unique zeros.

This completes the proof of Theorem 1.1.

\section{Acknowledgement}

The authors are grateful to the referee for a number of helpful suggestions to improve the paper.

\section{References}

[1] M. L. Fang and L. Zalcman, A note on normal families and sharing values, J. Aust. Math. Soc., 76 (2004), 151-150.

[2] Y. X. Gu, An normal criterion of meromorphic functions, Sci. Sinica, Math., Issue I (1979), 267-274.

[3] W. K. Hayman, Picard values of meromorphic functions and the its derivatives, Ann. Math., 70 (1959), 9-42.

[4] —_, Research problems in function theory, Athlone Press, London, 1967.

[5] J.M.Chang, Normality and quasinormality of zero-free meromorphic functions, Manuscript.

[6] Yuntong Li, Normal families of meromorphic functions with multiple zeros, J. Math. Anal. Appl., 381 (2011), 344-351.

[7] Yuntong Li and Yongxing Gu, On normal families of meromorphic functions, J. Math. Anal. Appl., 354 (2009), 421-425.

[8] J.M. Qi and T.Y. Zhu, Some normal families of meromorphic functions, Abstr. Appl. Anal., 2010 (2010), Arctile ID 147878, 4 pp.,doi:10.1155/2010/147878.

[9] Y. F. Wang and M. L. Fang, Picard values and normal families of meromorphic functions with multiple zeros, Acta Math. Sinica, Chinese Series, 41 (1998), 743-748.

[10] L. Zalcman, Normal families:new perspectives., Bull.Amer.Math.Soc.New Seri., 35 (1998), 215-230.

Department of Mathematics, Southwest University of Science and Technology, Mianyang 621010, P.R. China.

E-mail: luqiankuo1965@hotmail.com

Department of Material Science and Engineer, Southwest University of Science and Technology, Mianyang 621010, P.R. China.

E-mail: liaoql@swust.edu.cn 